\title{
Research on the Distribution Network's Single-phase Short-circuit Faults with Wavelet Transform
}

\author{
Hongyuan Huang, Yan Xu \\ Gunagdong Power Grid Corporation Foshan Power Supply Bureau,Guangdong, China \\ Automation Section,Creative Distribution Automation Co., Ltd,Beijing, China \\ e-mail: fszhd@vip.163.com,xuyan021@126.com
}

\begin{abstract}
Keywords: Single-phase Short-circuit Faults; Wavelet Transform; Wavelet Energy; Transient Zero Sequence Current
\end{abstract}

\begin{abstract}
According to the problem that the single phase grounding fault location is difficult to detect, a novel method of identifying fault line for single phase grounding fault is presented, based on the detailed analysis of the transient zero sequence network's fault characteristics in the small current neutral grounding power system. Experiments show that this method is immune to many factors such as the initial fault current switch angle, fault distance, transition resistance, power arc, the power distribution system operation mode and so on. It not only can detect the single phase high impedance grounding fault accurately which occurs at the terminal of transmission line, it also can detect the fault which occurs when the phase voltage is near zero.
\end{abstract}

\section{Introduction}

As is well known, there are several kinds of neutral grounding model according to the power actual circumstance and synthesis consideration by now, such as Neutral non-grounded way, Petersen-coil way, Neutral grounded way, Neutral non-grounded with resistance and so on[1][2]. The neutral grounding model is determined by the insulation level, power reliability and human safety and it also affects the fault current of grounding and the protection case. In distributions, because of its low-level voltage, its neutral point is usually disconnected to the ground in order to improve its reliability. As for this kind of distribution network, the single phase grounding fault location for transmission line is a problem in power system fault detection. There are many methods to deal with this problem, such as signal injection method, DESIR (Detection Selective par les Intensites Residuells), higher harmonic signals and so on [3][4]. But they are all ineffective.

In this paper, a novel method of identifying fault line for single phase grounding fault is presented, based on the detailed analysis of the transient zero sequence network's fault characteristics in the small current neutral grounding power system. At first, wavelet transform is applied to the data of every feeder's transient zero sequence current. Then the wavelet coefficients are employed to calculate the wavelet high frequency energy and the wavelet low frequency energy. In the end, the fault line of the single phase grounding fault is identified by comparing three phase line's maximum energy of the wavelet high or low frequency, because the energy spectrum features are different when the faults occur near the peek and zero point of the line-to-neutral voltage. Many simulations have been done and the results indicate that this method is immune to many factors such as the initial fault current switch angle, fault distance, transition resistance, power arc, the power distribution system operation mode and so on. It not only can detect the single phase high impedance grounding fault accurately which occurs at the terminal of transmission line, it also can detect the fault which occurs when the phase voltage is near zero.

\section{Analysis about the Fault Transient Characteristics of Zero Sequence Current}

In small current neutral grounding power system, the zero sequence network diagram of a single phase ground fault can be shown as Fig1 when there is a single phase ground fault. In this diagram, $\mathrm{K}$ is a switch. When it starts to operate, the neutral point is grounded via extinguisher. On the 
contrary, when it is closed, the neutral point is ungrounded. The overhead lines can be considered as an equivalent $\pi$ network. $L_{0 i}$ is the zero sequence line inductance, $R_{0 i}$ is the zero sequence line resistance, $C_{0 i}$ is the distributed capacitance, $U_{0}$ is the bus's zero sequence voltage, $L$ is the zero sequence inductance of the arc suppression coil, $R_{f 0}$ is the zero sequence transition resistance, $U_{f 0}$ is the voltage drop of the phantom power in the zero sequence network at the fault location. ${ }^{n}$ is the number of the outgoing feeder line.

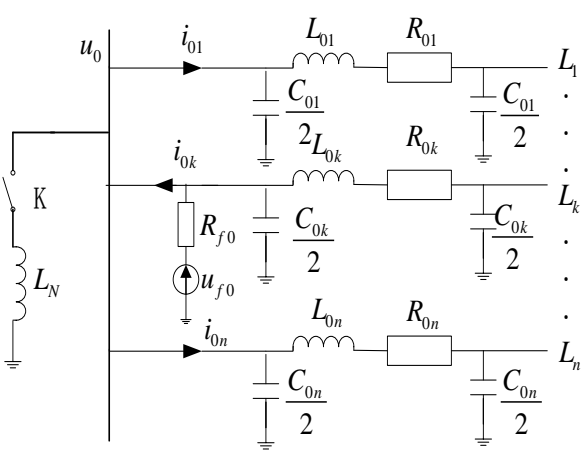

Fig.1. Zero sequence network diagram of a single phase ground fault

In the Fig.1, any feeder line’s zero sequence current can be described as (1) [5].

$$
R_{0 i} i_{0 i}+L_{0 i} \frac{d i_{0 i}}{d t}+\frac{1}{C_{0 i}} \int_{0}^{t} i_{0 i} d t=U_{0} \sin (\omega t+\varphi)
$$

When there is a single phase ground fault in the system, the total transient zero sequence current in the fault line is equal to the sum of the transient inductance current and the transient capacitance current. The total transient zero sequence current is described as (2):

$$
i_{0 k}=i_{L}+i_{C}
$$

As for the transient inductance current is described as type (3):

$$
i_{L}=i_{L d c}+i_{L s t}=I_{L m}\left[\cos \phi e^{t / \tau_{L}}-\cos (\omega t+\phi)\right]
$$

In the type: $i_{L d c}$ is the transient direct component of the inductance current; $i_{\text {Lst }}$ is the steady state fundamental frequency component of the inductance current; ${ }^{\phi}$ is the initial phase angle of phase voltage when there is a fault; ${ }^{I L m}$ is the amplitude of the inductance current; ${ }^{\tau_{L}}$ is the time constant of the inductance circuit.

The transient capacitance current can export as (4):

$$
i_{C}=i_{C o s}+i_{C S t}=I_{C m}\left[\left(\frac{\omega_{f}}{\omega} \sin \phi \sin t-\cos \phi \cos \omega_{f} t\right) e^{-\delta t}+\cos (\omega t+\phi)\right](4)
$$

In the type: $i_{C o s}$ is the transient component of free oscillation; $i_{C s t}$ is the steady state fundamental frequency component of the capacitance current; ${ }_{C m}$ is the amplitude of the capacitive current; $\delta$ is the decay factor of the free oscillation component; ${ }^{\omega_{f}}$ is the angular frequency of the transient free oscillation component.

If there is a single phase grounding fault, the transient inductance current and the transient capacitance current which decreased more slowly are generated in the fault lines. The transient 
inductance current usually is much less than the transient capacitance current and the extinguisher can't work very well in preliminary period of fault, so the zero sequence current's transient characteristics of the fault line and the non-faulted line are decided by the transient capacitance current if the fault occurs near the peak of the phase voltage. The relevant energy mainly focuses on high frequency (300-1500HZ) [6][7].. On the contrary, when the fault occurs near the zero-crossing point of the phase voltage, the total energy of transient zero sequence current in the non-faulted lines mainly focuses on low frequency $(0-50 \mathrm{HZ})$, because the transient capacitance current's free oscillation component is zero and the fundamental frequency component is large. The transient inductance current is composed by DC component and the fundamental frequency component, so the energy of the fault line mainly focuses on low frequency (0-50HZ) [5] [8].

Due to the excellent time-frequency characteristic, wavelet transform which is a multi-scale signal analysis method is suitable for analyzing transient and time-varying properties of nonstationary signals and has been well applied in recent years. When there is a single phase grounding fault in distribution network, the zero sequence current is non-stationary signal which is nonlinear and very complex. Wavelet transform is suitable for the extraction of the zero sequence current's transient characteristics, being of a multi-resolution feature [4][6].

The theory of multi-resolution analysis is the fundamental of the wavelet transform and it meets the demands of the two-scale equation.

$$
\begin{aligned}
& \varphi(t)=\sqrt{2} \sum_{n=-\infty}^{+\infty} h(n) \varphi(2 t-n) \\
& \psi(t)=\sqrt{2} \sum_{n=-\infty}^{+\infty} g(n) \varphi(2 t-n)
\end{aligned}
$$

In (5) and (6): $\varphi(t)$ is the scaling function; $\psi(t)$ is the wavelet function; ${ }^{h(n)}$ and ${ }^{g(n)}$ are the coefficients of the wavelet decomposition filter banks.

The discrete time signal is operated by up to a certain times by discrete wavelet transform. Then the wavelet coefficients high frequency component and the wavelet coefficients low frequency component are gained. The wavelet energy of single scale is the sum of squares of the wavelet coefficients. It is known that $\mathrm{db}$ series wavelet functions preserve many properties, such as orthogonality, compact support, much sensitive to irregular signals and so on. The db6 series wavelet functions are selected [7].

\section{Theory about fault line selection based on maximum of the wavelet energy}

The amplitude of transient zero sequence current in fault feeder is equal to the total amplitude of the transient zero sequence current in non-fault feeder. The wavelet energy is the sum of squares of the wavelet coefficients. In all, the accuracy of the fault selection is increased, because the difference of the fault judging quantity in fault feeder and non-fault feeder is increased by comparing every line's maxima wavelet energy [8]. In this paper, the fault line selection based on the maxima of the wavelet energy is introduced as follows.

1) The amplitude of transient zero sequence current in fault feeder is equal to the total amplitude of the transient zero sequence current in non-fault feeder. The wavelet energy is the sum of squares of the wavelet coefficients. In all, the accuracy of the fault selection is increased, because the difference of the fault judging quantity in fault feeder and non-fault feeder is increased by comparing every line's maxima wavelet energy [8]. In this paper, the fault line selection based on the maxima of the wavelet energy is introduced as follows.

2) Based on the db6, wavelet decomposition is employed to the zero sequence current of every outlet lines. Then the wavelet coefficients high frequency component and the wavelet coefficients low frequency component are applied to calculate the wavelet high frequency energy $E_{h}$ and the 
wavelet low frequency energy ${ }^{E_{l}}$ respectively.

3) When the minimum of the wavelet high frequency energy $E_{h}$ is less than the threshold $E_{\text {set }}$ which is set previously, it is considered that the fault occurs at the time when voltage is 0 and it is need to compare the wavelet low frequency energy $E_{l}$. On the contrary, it is need to compare the wavelet high frequency energy $E_{h}$. $E_{\text {set }}$ can be set based on the construction site and the detection accuracy of the device.

4) Every feeder's wavelet energy is compared and the feeder which contains the maximum wavelet energy is got. In the end, the maximum wavelet energy is compared to another feeder's

energy. When the maximum energy is greater than sun of another feeder's energy $\quad \begin{gathered}i=1 \\ i \neq j\end{gathered}$, the relevant feeder is the fault line. On the contrary, the bus is the fault line. The whole process can be shown in Fig 2.

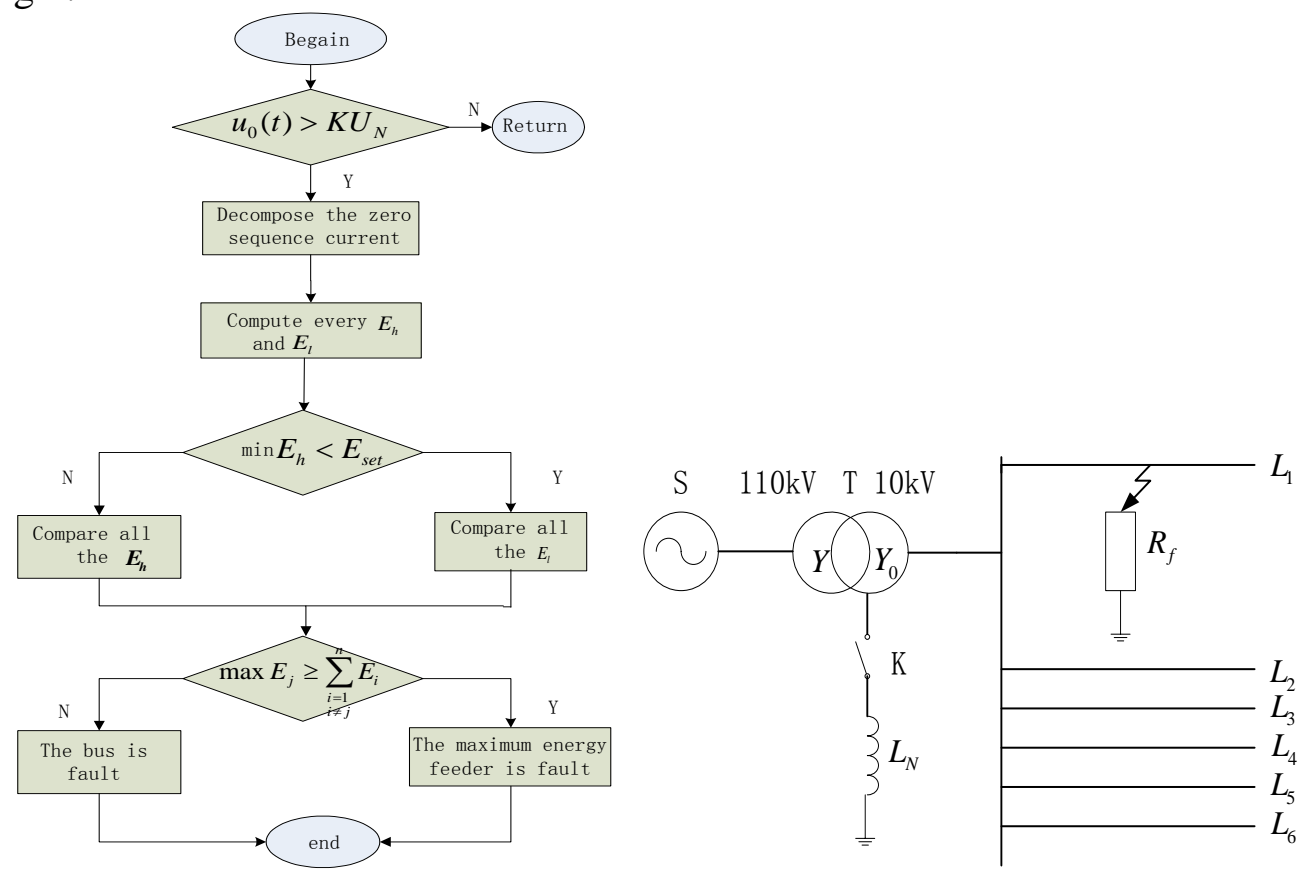

Fig.2. Course of the fault line selection Fig.3. Example of the fault line selection Simulation

\section{Test results}

The schematic of the single phase grounding fault is shown in Fig.3. This simulation system contains a substation which has 6 lines of $110 \mathrm{KV} / 10 \mathrm{KV}$. The connection of the transformer is $Y / Y_{0}$. The line parameters are as follows:

$$
r_{1}=0.17 \Omega / \mathrm{km}, \quad l_{1}=0.38 \times 10^{-3} \mathrm{H} / \mathrm{km}, \quad c_{1}=9.69 \times 10^{-9} \mathrm{~F} / \mathrm{km}, \quad r_{0}=0.23 \Omega / \mathrm{km}, \quad l_{0}=1.72 \times 10^{-3} \mathrm{H} / \mathrm{km}, \quad c_{0}=6 \times 10^{-9} \mathrm{~F} / \mathrm{km} \text { 。 }
$$

The parameters of every line are as follows: $L_{1}=3 \mathrm{~km}, L_{2}=6 \mathrm{~km}, L_{3}=9 \mathrm{~km}, L_{4}=12 \mathrm{~km}$, $L_{5}=15 \mathrm{~km}, L_{6}=20 \mathrm{~km}$, and theirs equivalent load are $Z_{L}=(400+j 20) \Omega$. The sample frequency is 10KHZ. The wavelet high frequency energy $E_{h}$ and the wavelet low frequency energy $E_{l}$ is calculated based on the $\mathrm{d} 4$ coefficient and a5 coefficient respectively. The time window is 10 milliseconds. $E_{h}$ is set as 10 .

When the $\mathrm{K}$ is on, the neutral point is grounded via the Petersen-coil and the formula for $L_{N}$ is as (7):

$$
L_{N}=\frac{1 \times 10^{6}}{3(1+v \%)\left(2 \pi f_{N}\right)^{2} l c_{0}}
$$


In (7): ${ }^{l}$ is the total length of the lines; $f_{N}$ is $50 \mathrm{HZ} ;{ }^{c_{0}}$ is the zero sequence capacitor; $v$ is the degree of detuning, $v=\left(I_{c}-I_{L}\right) / I_{c}$.

The PSCAD/EMTP is applied to simulate the system. Many simulations are done and the results are analyzed with Matlab. The results are as follows in Fig.4.

When the fault happens at the end of the feeder 4 , the fault angle is $90 \%$, the grounding resistance is $100 \Omega$, the overcompensation degree is $6 \%$, the fault occurs at $0.32 \mathrm{~S}$, the simulation of all the feeder's zero current sequence is displayed in Fig. 5 and the relevant wavelet energy $E_{h}$ is in Fig.6. From the Fig.6, it shows that the fault is in line 4 and the result is right.

\begin{tabular}{|c|c|c|c|c|c|c|c|c|c|c|c|}
\hline$L_{1}$ & $R_{f} / \Omega \theta$ & $9 / 0^{\circ}$ & $\left.{ }^{\circ}\right)$ & $X_{f} / \mathrm{km}$ & & relet & energy $\boldsymbol{E}$ & $E_{i} \quad i=16$ & & & result \\
\hline \multirow{3}{*}{1} & 5 & 0 & over $8 \%$ & 1 & 374 & 20 & 17 & 15 & 13 & 11 & right \\
\hline & 100 & $60 \mathrm{u}$ & unground & 1.5 & 935 & 45 & 43 & 42 & 31 & 28 & right \\
\hline & 1000 & $90 \mathrm{c}$ & under $8 \%$ & 2 & $\begin{array}{c}224 \\
8\end{array}$ & 90 & 91 & 93 & 87 & 88 & right \\
\hline \multirow{3}{*}{4} & 5 & 90 & unground & 6 & 755 & 654 & 604 & 14268 & 476 & 404 & right \\
\hline & 100 & 30 & over $15 \%$ & 2 & 31 & 35 & 30 & 757 & 31 & 25 & right \\
\hline & 1000 & $\begin{array}{ll}0 & \mathrm{u}\end{array}$ & under $18 \%$ & 8 & 88 & 83 & 82 & $\begin{array}{c}215 \\
5\end{array}$ & 80 & 76 & right \\
\hline \multirow{3}{*}{6} & 5 & $45 \mathrm{u}$ & under $4 \%$ & 12 & 27 & 23 & 22 & 21 & 14 & 514 & right \\
\hline & 100 & 0 & unground & 10 & $\begin{array}{c}577 \\
6\end{array}$ & $\begin{array}{c}574 \\
9\end{array}$ & $\begin{array}{c}572 \\
1\end{array}$ & & $\begin{array}{c}565 \\
0\end{array}$ & $\begin{array}{c}142867 \\
165\end{array}$ & $\begin{array}{l}\text { right } \\
\text { right }\end{array}$ \\
\hline & 1000 & 90 & over $20 \%$ & 4 & 65 & 66 & 72 & 58 & 70 & 5 & right \\
\hline \multirow{3}{*}{ Bus } & 5 & 0 & over 5\% & 0 & 35 & 31 & 26 & 22 & 18 & 14 & right \\
\hline & 100 & 90 & unground & 0 & 89 & 97 & 92 & 80 & 72 & 60 & right \\
\hline & 1000 & 60 & under $9 \%$ & 60 & 65 & 60 & 59 & 75 & 68 & 55 & right \\
\hline
\end{tabular}

Fig.4. Results of the fault line selection

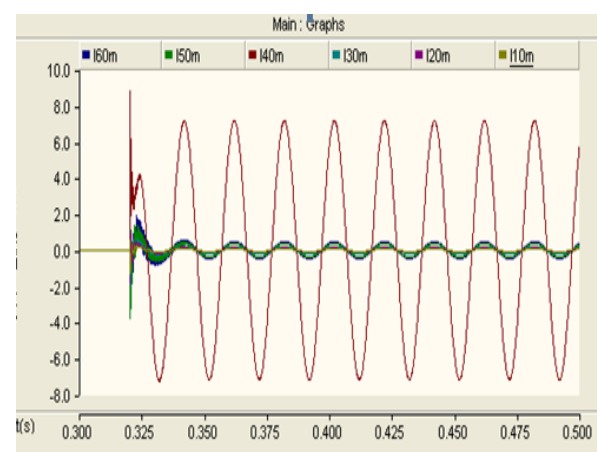

Fig.5. Results of the fault line selection

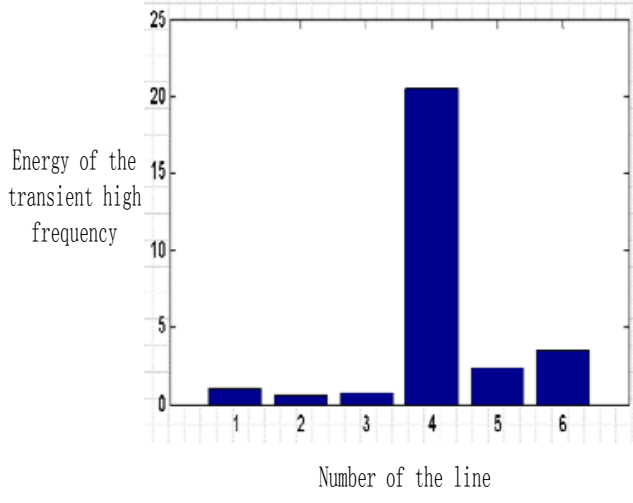

Fig.6. Results of the fault line selection

\section{Conclusion}

Based on the analysis of the transient zero sequence network's fault characteristics in the small current neutral grounding power system, it not only can detect the single phase high impedance grounding fault accurately which occurs at the terminal of transmission line, it also can detect the fault which occurs when the phase voltage is near zero.

\section{Acknowledgment}

It is a project supported by the High-Tech Research and Development Program of China (863 Program) (2011AA05A114). 


\section{References}

[1] S. Curcic, C. S. Ozveren, L. Crowe, EK. L. Lo. Electric power distribution networkretoration : a survey of papers and a review of restoration problem[J]. Electric PowerSystems Research 35(1996) : 73〜86.

[2] T Ananthapadmanabha. A D Kulakami. A S G rao. J G Char and K R Ran, kParthasarathy. Knowledge_-based methodology for intelligent sequence switching, fault identification and service restoration of distribution system[J ]. Electrical Power\& Energy Systems, V01. 19. No. 2. PP. 119 124. 1997.

[3] Zhou Deng-deng, Liu Zhi-gang, Hu Fei, Bai Wei-li. A new method for fault line selection based on wavelet de-noising and transient current energy grouping comparison in ineffective grounding system [J]. Power System Protection and Control, 201038 (7): 22-28.

[4] WANG Yao-nan, HUO Bai-lin, WANG Hui, et al. A New Criterion for Earth Fault Line Selection Based on Wavelet Packets in Small Current Neutral Grounding System[J]. Proceedings of the CSEE, 2004, 24(6) : 54-58.

[5] XUE Yong-duan, XU Bing-yin, et al. Earth Fault Protection and Monitoring Technology Using Transient Signal in Non-solid Earthed Network[Z]. Zibo : Ke Hui Electric Power, 2005.

[6] SANG Zai-zhong, ZHANG Hui-fen, PAN Zhen-cun. Singal Phase Grounding Fault Protection by Injecting Currents in Ineffective Grounding Systems[J]. Automation of Electric Power Systems, 1996, 20 (2) : 11-13.

[7] ZHANG Li-hua, XU Wen-li. A New Algorithm of Fault Line Detection in Small Current Neutral Grounding System[J]. Journal of Tsinghua University, 1998，38（9） : 74-76.

[8] CHEN Kui, TANG Yi. Study on Faulty Line Detection of Single Phase to Ground Fault in Arc Suppression Coil Grounded System[J]. Relay, 2005, 33 (16) : 5-9. 